Aus dem Laboratorium der II. Frauenklinik Wertheim in Wien.

Laboratoriumsvorstand: Prof. Schottlaender.

\title{
Cystisches Sarkom des Beckenperitoneums etwa vier Jahre nach Sarkom des Uterus.
}

\author{
Von \\ Dr. Ephraim Goldenstein. \\ (Hierzu Tafel XI.)
}

Die im Folgenden zu erörternden Besonderheiten des Falles rechtfertigen, wie ich hoffe, die nachstehende Veröffentlichung:

Am 11. IV. 1910 wurde Frau F. S., 43 Jahre alt, bei uns aufgenommen, die wegen heftiger, seit 6 Wochen eingetretener Schmerzen in der linken Unterbauchseite und Zunahme des Leibesumfanges klinische Hülfe aufsuchte. Patientin, früher stets gesund, hat sieben Mal geboren, zuletzt vor 8 Jahren. Die in ihrem 20. Jahr eingetretene Periode war bis zur letzten Schwangerschaft normal. Im Ansehluss an die letzte Geburt stellten sich unregelmässige Blutungen ein, die im Juni 1905 eine Auskratzung notwendig machten. Am 22. September 1906 begannen die Blutungen, die inzwischen aufgehört hatten, von Neuem; es gesellten sich Kreuzschmerzen hinzu und Patientin wandte sich am 1. X. 1906 an die Klinik Chrobak, um von ihren Beschwerden befreit zu werden.

Die Untersuchung ergab damals (laut Krankengeschichte) folgenden Befund: Abdomen im Thoraxniveau. Der Uterus, dessen Fundus in der Höhe der Symphyse zu tasten ist, erscheint im Gebiet des linken Hornes dicker und breiter als rechts. Parametrien frei. Adnexe bis auf leichte Druckempfindlichkeit des linken Eierstocks ohne Besonderheiten. Die Diagnose lautete auf Metritis chronica. - Da die konservative Therapie versagte, wurde im October 1906 die vaginale Totalexstirpation des Uterus unter Belassung der beiderseitigen Adnexe vorgenommen. Bei der Operation erwies sich das linke Ovarium als kleincystisch degenerirt; es wurde mit einem Skalpell mehrmals angestochen und der Inhalt entleert. Das rechte zeigte keine Besonderheiten.

Der - Ieider nicht aufbewahrte - Uterus erwies sich laut Bericht im Gebiet des linken Horns vergrössert, verdickt und zeigte auf dem Durchschnitt in dieser Gegend einen etwa wallnussgrossen, gelappten und an der Oberfläche stellenweise geschwürig zerfallenen, zum Teil polypös in das Cavum uteri hineinragenden Tumor. Letzterer wurde makroskopisch für ein Myom gehalten; doch ergab sich bei der mikroskopischen Untersuchung, dass es sich um ein Sarkom handelte. Ein Präparat aus der damaligen Zeit ist noch in unserem Besitz und soll später genauer besprochen werden. 
Der klinische Befund bei der neuerlichen Untersuchung am 11. IV. 1910 war folgender: Mittelgrosse, stark gebaute Frau. Hautdecken und sichtbare Schleimhäute normal: Ernährumgszustand mittelgut; Lungen und Herz ohne Besonderheiten. Gynäkologischer Status: die Scheide endet blind. Der Uterus fehlt. Die hintere Scheidenwand ist durch einen unregelmässig gestalteten, im Becken fixierten, zumeist derben, glattwandigen Tumor vorgewölbt. Derselbe lässt sich, da die Patientin stark spannt, nach oben nicht deutlich abgrenzen; links erstreckt er sich bis an die Beckenwand und an dieser aufwärts. Adnexe nicht zu tasten.

Bei der am 18. April vorgenommenen Laparotomie erwies sich der Tumor aus teils kompakten, teils cystischen Abschnitten zusammengesetzt. Er geht vom linken Eierstock aus. Im Becken wenig blutig gefärbte Flüssigkeit. Im Douglas sehr reichliche multiple Excrescenzen, die daselbst mit dem Peritoneum verwachsen sind. Auch mit dem Parietalperitoneum ist eine starke Verlötung nachzuweisen, so dass sich die Auslösung schwierig gestaltet und mehrfach Geschwulstreste am Peritoneum zurückbleiben, auch deshalb, weil die soliden Partien bei der Berihrung leicht zerbröckeln. Der rechte Eierstock ist nicht zu. isolieren. Die rechte Tube ist cystisch erweitert und verschlossen, die linke nicht kenntlich. Die Abtastung des Magens ergibt keine Veränderungen.

Makroskopischer Befund. Eine Reihe einzelner Gewebsstücke von zum Teil sehr eigentümlichem Aussehen. Eines derselben ist auf Fig. 1 dargestellt. Man erkennt ohne Weiteres, dass solide und cystische Partien in buntem Wechsel vorhanden sind. Bei ersteren, bei welchen vielfach die ausgesprochene glatte, bald mehr gelblich, bald rötlich gefärbte Oberfläche auffällt, handelt es sich entweder um mehr walzenförmige weiche oder um strangförmige derbere Bildungen, die häufig wiederum sekundäre, solide, öfters mit fadendïnnem Stiel aufsitzende Knötchen tragen. Auf dem Durchschnitt erscheint das solide, in seinem Aufbau an eines der grossen parenchymatösen Organe, etwa die Leber, erinnernde Gewebe ohne Zeichnung. - Die Färbung ist die gleiche wie an der Oberfläche.

Die Cysten in ihrer Grösse zwischen einem Stecknadelkopf und einer Pflaume wechselnd liegen fast ausschliesslich oberflächlich und sind ziemlich dünnwandig, dabei prall gespannt; bei der Durchschneidung entleeren sie eine seröse Flüssigkeit. Deutlich schleimiger Inhalt wurde nicht gefunden.

Ausser den oben beschriebenen Gewebsstücken, von denen 5-6 vorhanden waren, die alle ungefähr denselben Bau besassen - das grösste ist abgebildet (Fig. 1) - gelangte noch zur. Untersuchung: eine Membran mit zahlreichen, vielfach gestielten Anhängen versehen, ferner ein $2^{1 / 2} \mathrm{~cm}$ langes Tubenstück mit deutlichem Fimbrienende fast ohne Ligamentgewebe. Von letzterem war nur am medialen Ende ein kleiner Rest vorhanden, dem ein ohne Weiteres kenntliches Eierstockstückchen anhaftete. Auch hier wiederum, oberflächlich gelegen, eine etwa kirschgrosse Cyste von der geschilderten Beschaffenheit.

Mikroskopischer Befund: Formolhärtung, Paraffineinbettung, Hämatoxylin-Eosin- sowie van Gieson- und Thioninfärbung.

I. Bei dem grossen auf Fig. 1 dargestellten Stück ergab sich derselbe Befund, wie bei den erwähnten anderen kleineren. Wir haben hier nur gesondert der soliden und der cystischen Anteile zu gedenken.

a) Die soliden Anteile bestehen durchweg aus kompakten, diffus 
ausgebreiteten Geschwulstherden, welche zumeist runde, selten längliche, kleine, ziemlich gleich grosse Zellen mit dunklen chromatinreichen Kernen (vergl. Fig. 3, 4) enthalten. Die Zellen, schlecht abgegrenzt, übertreffen Lymphocyten kaum an Grösse. Mehrere Kerne in einer Zelle sind nicht nachweisbar. Mitosen fehlen. Zwischen den Zellen ist häufig deutliche zartkörnige Intercellularsubstanz nachzuweisen, ferner finden sich recht zahlreiche, mitunter erweiterte Blutgefässe, deren Endothel gequollen und gewuchert erscheint, Manche Gefässe sind thrombosiert und hyalinwandig. Stellenweise ist reichlicheres Zwischengewebe vorhanden, das hyalin degeneriert erscheint. Weiter sind inmitten der Geschwulstherde mitunter Blutergüsse zu konstatieren, welche die Tumorzellen zerstört haben; es finden sich hier auchAnsammlungen von Lymphocyten. Geschwulstfreies Grundgewebe fehlt. An der Oberfläche der soliden Partien mitunter niedriges Epithel.

b) Cystische Anteile. Die verschieden grossen Cysten bieten insofern verschiedene Bilder, als die Hohlräume verschiedene Grösse und auch etwas verschiedene Gestalt besitzen. Ueber die Grösse siehe oben. Die Gestalt ist zumeist rund; bisweilen finden sich unregelmässige Buchten. Die Räume, von Geschwulstzellen umgeben, sind teils leer, häufiger mit homogenen, eosingefärbten. Massen gefïllt. Bei Thioninfärbung nehmen die homogenen Massen einen leicht violetten Farbenton an. Selten darin einige Lymphocyten, keine roten Blutkörperchen. Die Hohlräume besitzen keine Eigenwand - das umgebende Lager von Tumorzellen ist dadurch charakterisiert, dass letztere concentrisch und hier mehr langgestreckt, erstere umgeben. Häufig 2 Zonen: eine äussere lockere und eine innere kompaktere Schicht. Bei genauer Betrachtung ergibt sich, dass mitunter Geschwulstparenchym in die Cyste vorragt. Endothel ist nirgends nachweisbar. Es finden sich keine erweiterten Lymphräume in der Nachbarschaft, dagegen Uebergänge zwischen den oben beschriebenen, in Zerstörung begriffenen Geschwulstpartien und den runden Hohlräumen.

c) Noch bleibt des histologischen Bildes der feinen, auf Figur 1 deutlich erkennbaren bandförmigen Ausläufer zu gedenken. Es zeigt sich, dass dieselben aus kleinen blutgefülltten Gefässen bestehen, die sich weiterhin verzweigen und von zahlreichen Sarkomzellen eingescheidet erscheinen. Da, wo die Stränge in solide Partien übergehen, finden sich wieder die vorhin beschriebenen kompakten Zellanhäufungen ohne Cysten.

II. Eine Reihe von Schnitten durch das membranöse Stück ergibt Folgendes: Die Membran erweist sich als aus mässig derbem Bindegewebe bestehend, das etwa den Charakter von Peritonealgewebe besitzt, und auch oberflächlich von typischem Peritonealendothel überzogen erscheint. Fett, Muskulatur und Nerven hier nicht nachweisbar. An der inneren Oberfläche zeigen sich wieder solide und kleine cystische Sarkompartien, wie sie unter I. beschrieben worden sind. Doch fallen am Rande der Membran Hohlräume anderen Baues auf, die in Fig. 2 abgebildet sind. Die in weiteren Schnitten geschlossenen Räume sind hier bis auf einen offen (Schnittrichtung). Diese Cysten unterscheiden sich von den früheren dadurch, dass sich a) im Hohlraum Sarkommassen befinden, b) an seiner Wand verschiedentlich sicheres Endothel zu Tage tritt, welches die Feststellung der Cysten als Gefässe ermöglicht.

Die beiden kleineren Räume ohne eigene Wandung müssen als Lymphgefässe angesprochen werden. Der grösste Raum ist nicht ein- 
heitlich; der mit homogenen Massen gefiillte, in dem Bilde rechts liegende, welcher Lymphocyten und rote Blutkörperchen enthält, entpuppt sich infolge des Vorhandenseins einer eigenen Wandung und des Nachweises von Vasa vasorum als Vene, die offenbar auf der linken Seite durch Sarkom, vielleicht ausserdem auch noch artefiziell zerstört worden ist. Bei dem grösseren übrig bleibenden linken Raum fehlen die genannte Characteristica der Wandung, wir müssen ihn als Lymphraum auffassen. Von Interesse ist nun der Umstand, dass mit absoluter Sicherheit an der Oberfläche aller Sarkomherde ein fast vollständig kontinuierlicher Endothelbelag nachgewiesen werden konnte; der grösste Herd wird durch den Belag von dem Venenlumen getrennt. Bei starker Vergrösserung zeigt sich das Endothel im Allgemeinen völlig typisch gebaut; stellenweise sind kleine Lücken vorhanden, stellenweise ist es etwas rerdickt, erhöht (kubisch). Mehrschichtung fehlt. Mitosen sind. nicht zu sehen. Die an das Endothel angrenzenden Sarkompartien erscheinen lockerer als die davon entfernter liegenden und als das Centrum (vergl. Fig. 4) der Herde und unterscheiden sich dadurch auch von den vorhin beschriebenen. In dem mittelgrossen Herde (Fig. 2) ist nun weiter schon bei schwacher Vergrösserung ein aus Endothel bestehender Streifen zu konstatieren, der ihn in zwei Abschnitte sondert und in Fig. 3 bei stärkerer Vergrösserung dargestellt ist. In Fig. 4 ist eine Strecke der endothelbekleideten Gefässwand mit gegenüber und frei liegendem, gleichfalls endothelbekleidetem Sarkomparenchym abgebildet. Mit Hilfe von Serienschnitten liess sich ermitteln, dass an Stelle des Endothelstreifens zunächst ein nach beiden Seiten ron Endothel bekleideter schmaler Spalt auftritt, wobei das Endothel wiederum den Geschwulstmassen dicht aufsitzt; weiter, dass die beiden Geschwulstherde sich voneinander trennen und schliesslich an die Gefässwand rücken (Fig. 5), wobei sie von dem Endothel letzterer kontinuierlich ïberzogen werden. In weiteren Schnitten durch die Membran fand sich. auch eine grössere blutgefülllte Vene mit einem Sarkomnest, das auch frei, ohne Zusammenhang mit der Gefässwand lag und kontinuierlich von Endothel überzogen war.

III. Die Untersuchung der Tube mit anhaftendem Ligamentgewebe und 0varialrest (vergl. den makroskopischen Befund) ergab) Folgendes:

Die Tube (Querschnitte) zeigt normalen Faltenban. Das Stroma der Falten, von spärlichen Lymphocyten durchsetzt, enthält erweiterte Gefässe. Das Epithel, typisch gebaut, ist auf der Höhe der Falten stellenweise zu Grunde gegangen. Im Lumen keine entzündlichen Infiltrate. Nirgends Geschwulstbildung.

Das kleine Stiick des der Tube anhaftenden hyperämischen Ligamentgewebes enthält wiederum eine Reihe grösserer und kleinerer Sarkomherde, die zum Teil beginnenden Zerfall erkennen lassen. Einige der letzteren sind zweifellosen erweiterten Lymphgefässen eingelagert. Auch hier erwiesen sich die frei im Lumen befindlichen Gesehwulstmassen auf grössere Strecken hinaus mit einem deutlich zu differenzierenden Endothelüberzug bekleidet, während die Gefässwand selbst nur an einzelnen Stellen einen solchen erkennen lässt.

Was das in Serien aufgeschnittene Ovarialstü ck (Fig. 6) anbetrifft, so zeigt es sich auch bei genauester Untersuchung geschwulstfrei. Es ist wesentlich nur Rindenstroma nachweislich, das derb und fast völlig 
follikellos ist. Nur ein Follikel mit zweischichtigem Epithel ist vorhanden, ferner ein cystisches Corpus luteum und eine Reihe von weissen Körpern. Die Gefässe sind erweitert, zum Teil hyalin. An der Oberfläche des anhaftenden Ligamentgewebes findet sich streckenweise deutliches kubisches Endothel. Wie Fig. 6 lehrt, sitzt dem Eierstock an einer Stelle eine rundliche Cyste auf, deren Grösse diejenige des Eierstockabschnittes ganz bedeutend iibertrifft. Die Cystenwand ist teils glatt, teils gebuchtet, diün, streckenweise hyalin und entzündlich infiltriert; sie erscheint als die direkte Fortsetzung eines an der Oberfläche des Ovariums gelegenen, entsprechend zusammengesetzten Gewebsstreifens. Innen erweist sich die Cystenwand fast überall mit einem zusammenhängenden Endothelsaum austapezirt. Im Hohlraum liegen verschiedentlich eosingefärbte Massen wie oben beschrieben, die indessen bei Schleimfärbung (Thionin) keinen violetten, sondern einen blauen Farbenton annehmen. In dem Cystenhohlraum weiter an 2 Stellen Sarkomherde, die denselben Bau, wie die früher beschriebenen zeigen. Der kleinere Herd sitzt der Innenwand anf und erweist sich wiederum in einer Reihe von aufeinanderfolgenden Schnitten mit deutlichem Endothel bekleidet, das in anderen fehlt. In den Anfangsschnitten lässt sich das Endothel der Wand in direkter Kontinuität auf den Geschwulstherd verfolgen. Während nun aber diese anfänglichen und die letzten der gefertigten 165 Schnitte ausser dem freiliegenden grossen nur den erwähnten kleineren, der Wand anliegenden Herd enthalten, zeigen die mittleren $(20-60)$ noch einen oder zwei kleine Herde, die, dem inneren kleinen gegenüber, der Wand aussen aufsitzen und diese verschiedentlich mit Ausläufern durchsetzen. Ein tieferes Eindringen, ein Durchbruch und eine Vereinigung des äusseren und des inneren Herdes konnte, obgleich das Gebiet der betreffenden Herde völlig aufgeschnitten worden ist, nicht nachgewiesen werden; ein Teil der Schnitte ist beim Schneiden zerrissen worden. An einer Verbindung ist indessen um so weniger zu zweifeln, da schon in den vorhandenen Schnitten die Annäherung der Herde beträchtlicher und auch von dem inneren ein in die Wand reichender Fortsatz allmählich immer deutlicher wird.

Was den grösseren (in der Fig. 6 unten liegenden) Herd anbetrifft, so wird er immer kleiner und verschwindet schliesslich, ohne auf irgend einem Schnitte mit der Cystenwand in Verbindung zu treten.

IV. Noch muss einer Cyste gedacht werden, die gesondert untersucht wurde. Ihre Wandung besteht aus geschwulstfreiem Bindegewebe mit glatter Muskulatur und ist mit kubischem Epithel ausgekleidet. An einer Stelle zeigt sie einen papillären, entsprechend bekleideten Vorsprung. Dass es sich um die Tube handelt wird ohne Weiteres dadurch klar, dass an einer anderen Stelle noch deutliche zum Teil miteinander verklebte Schleimhantfalten sich finden. Das Stroma ist hyperämisch, stellenweise entzündlich infiltriert, nirgends sind Geschwulstherde nachzuweisen.

\section{Epikrise.}

Ueberblicken wir unsere Befunde bei dem beschriebenen Fall, so ergibt sich, dass bei einer 43 jährigen Patientin, fast 4 Jahre nach Entfernung des Uterus samt einem sarkomatösen Funduspolypen, gelegentlich einer durch grosse Beschwerden veranlassten 
Laparotomie, zahlreiche sarkomatöse Geschwulstmassen im kleinen Becken gefunden wurden. Es fragt sich nun zunächst, in welchem gegenseitigen Verhältnis die verschieden lokalisierten Geschwülste gestanden haben. Der cinzig von früher her in unseren Händen befindliche Schnitt durch den Uteruspolypen ergiebt, dass diffus ausgebreitet kleine, vorwiegend runde Geschwulstzellen vorhanden sind, in deren Gebiet sich stellenweise Blutergüsse bemerkbar machen. An der Oberfläche ist ein diskontinuierlicher Saum von kubischcylindrischem Deckepithel zu sehen.

Dass es sich somit beide Male um ein im Wesentlichen durchaus gleichartiges, vornehmlich kleinzelliges Rundzellensarkom handelt, steht ausser Zweifel und es ist daher am nüchsten liegend an ein direktes Abhängigkeitsverhältnis in dem Sinne zu denken, dass ein primäres Sarkom des Uterus entstanden war, welches sekundär zur Erkrankung des Beckenbindegewebes bzw. Peritoneums geführt hat. Leider steht uns das makroskopische Präparat nicht mehr zur Verfügung - der erwähnte Schnitt weist nur infolge der Anwesenheit des Epithelsaumes darauf hin, dass es sich um eine in den inneren Schichten des Uterus lokalisierte Geschwulst gehandelt hat. Da die Stelle, welcher der Schnitt entstammt, unbekannt ist, so kommt neben einem Schleimhaut- auch ein submuköses Myosarkom oder Myoma malignum in Betracht, obwohl Muskelzellen fehlen. Wir sind im Uebrigen lediglich auf Vermutungen angewiesen. Es könnte einmal eine kontinuierliche Durchwachsung der Uterussubstanz mit Dissemination von Tumormassen im Bauchraum stattgefunden oder aber es könnte sich die Erkrankung auf dem Wege retrograder Lymph- oder Blutgefässmetastasen von dem Polypen aus verbreitet haben. Wenn wir bedenken, dass retrograde Metastasierung [v. Recklinghausen ${ }^{1}$ )] jedenfalls kein allzu häufiges Ereignis ist, ja.sogar von manchen Pathologen [Ribbert²) - auf dem Lymphwege, Borrmann ${ }^{3}$ ) - auf dem Blut- und Lymphwege] geleugnet wird; wenn wir weiter bedenken, dass speciell die polypösen Uterussarkome im Ganzen spät Metastasen setzen [Kaufmannt)], so dürfte der erstgenannte Modus der Weiterverbreitung wohl als wahrschein-

1) v. Recklinghausen, Ueber die venöse Embolie und den retrograden Transport etc. Virchows Archiv. 1885.

2) Ribbert, Die Geschwulstlehre.

3) Borrmann, Ein diffuses Riesenzellensarkom der Cervix uteri eto. Zeitschr. f. Geb. u. Gyn. Bd. 43.

4) Kaufmann, Lehrbuch d. spez. Pathologie. 
licher angesehen werden, es sei denn, dass wir das im Beckenraum angetroffene Sarkom als unabhängig von demjenigen des Uterus entstanden ansehen wollten, wofür indessen trotz des jetzt wohl sichergestellten Vorkommens derartiger multipler Tumorbildung hier nicht der geringste Anhaltspunkt besteht, ja, wogegen bis zu einem gewissen Grade die sofort in die Augen springende Aehnlichkeit des Geschwulstgewebes in den verschiedenen befallenen Organen spricht. Es ist kaum nötig zu erwähnen, dass ein primäres Sarkom eines anderen Bauchorgans, von dem aus, wie bekannt [Glockner ${ }^{1}$ ), Kraus ${ }^{2}$, Schlagenhaufer ${ }^{3}$ ), Stickel ${ }^{4}$ ), Brunner ${ }^{5}$ ), Tilp ${ }^{6}$ ) u. a.] im Gegensatz zum Uterus sehr viel häufiger Metastasen im Beckenraum, besonders im Douglas, ausgehen, hier infolge der genauen Untersuchung und der Besichtigung der Bauchorgane bei der Operation nicht in Frage kommt; auch ein primäres Sarkom der Brustorgane kann ausgeschlossen werden. - Wie hervorgehoben wurde, war abgesehen von der Cystenbildung das organähnliche Aussehen der einzelnen Geschwulststücke auffallend, ferner die absolut glatte, beinahe glänzende Oberfläche. Aus letzterem Umstande musste der Schluss gezogen werden, dass die Geschwulstmassen von Peritoneum überzogen seien, was sich auch mikroskopisch zum Teil durch den exakten Nachweis von Peritonealepithel bestätigte.

In der Krankengeschichte ist die Rede von doppelseitigem Ovarialsarkom; die linken Adnexe wurden ausserdem als cystisch angesprochen; man nahm an, dass das linke Ovarium das von der sekundären Erkrankung mehr befallene sei und dass von ihm ein Strang, der Rundung des Douglasraumes folgend, nach dem rechten hinüberziehe. Wir haben (siehe oben) nur einen Teil der Adnexe der einen Seite, ferner einen Rest der anderseitigen Tube konstatieren können. Die Tuben waren, auch in ihrem Ligamentgewebe, völlig frei von Geschwulstmassen; die eine, bei der das zugehörige Ovarium

1) Glockner, Ueber sekundäres Ovarialcarcinom. Dieses Archiv. Bd.72.

2) Kraus, E., Ueber das Zustandekommen der Krebsmetastasen im Ovarium etc. Monatsschr. f. Geb. u. Gyn. Bd. XIV.

3) Schlagenhaufer, Ueber das metastatische Ovarialearcinom etc. Monatsschrift f. Geb. u. Gyn. Bd. XV.

4) Sticke1, Ueber doppelseitige metastatische Ovarialcarcinome. Dieses Archiv. Bd. 79.

5) Brunner, Doppelseitiges metastatisches Ovarialcarcinom etc. Zeitschr. f. Geb. u. Gyn. Bd. 61 .

6) Tilp, Zur Kenntnis der Implantationscarcinome im Abdomen. Berliner klin. Wochenscbr. 1908. No. 25. 
fehlte, zeigte die Erscheinungen einer ziemlich weit fortgeschrittenen Salpingitis cyst. cat. (Hydrosalpinx); es konnte die Tubennatur (siehe oben) nur an einer noch übrig gebliebenen vorspringenden Schleimhautzotte festgestellt werden. Die andere Tube zeigte leichte Entzündung. Von dem zugehörigen Ovarium (Fig. 6) war nur ein kleinster Rest, in dem chronische Ernährungsstörungen nachweisbar waren, zu finden. Auch im Eierstocksgewebe selbst fehlte jede Geschwulstbildung, während eine solche in dem umgebenden Ligamentgewebe deutlich war. Unter diesen Umständen ist die klinische Diagnose einer (sekundären) Ovarialerkrankung anatomisch-histologisch nicht mit Sicherheit zu erhärten. Wenn auch natürlich nicht ausgeschlossen werden kann, dass der Eierstock der einen Seite ganz, der der anderen zum grösseren Teil durch ein fortschreitendes Wachstum des Sarkoms im umgebenden Gewebe zerstört worden ist, so müssen wir doch Bedenken tragen, diese Auffassung ohne Weiteres zu acceptieren. Es ist zum Mindesten sehr auffallend, dass das kleine vorhandene Ovarialstück in seinem Gewebe so vollständig von der Erkrankung verschont geblieben ist. Es darf meiner Ansicht nach auch mit der Möglichkeit gerechnet werden, dass bei der Operation Ovarialgewebe im Banchraum zurückgelassen worden ist, da nicht nur die richtige Bewertung der zahlreichen einzeln und unvollständig entfernten Tumormassen, sondern auch die Orientierung während der Operation auf grösste Schwierigkeiten gestossen sein muss.

Das beschriebene Sarkomgewebe ist nun aus verschiedenen Gründen von Interesse. Dass es sich in dem Fall tatsächlich um ein wesentlich klein- und rund-, zum Teil spindelzelliges Sarkom handelt, bei dem die deutlich nachweisbare Zwischensubstanz, sowie die diffuse Anordnung der Elemente die Differentialdiagnose gegen Carcinom und Endotheliom sichert, kann keinen Augenblick zweifelhaft sein.

Die genauere Beobachtung ergab nun nach zweierlei Richtungen wichtige Befunde. Erstens einmal konnte dargetan werden, dass an manchen Stellen eine eigenartige Beziehung der Sarkomzellen zu Blutgefässen vorhanden. war. Man erinnert sich der lang ausgezogenen bandartigen Stränge (Fig. 1), die ausser der Cystenbildung manchen der entfernten Gewebsstücke ein so eigenartiges Aussehen verliehen. Die mikroskopische Untersuchung ergab bei einem Längsschnitt im Centrum der von diesen Strängen ausgehenden feinsten Fäden, die an ihrem äusseren Ende mit einem 
kleinen Sarkomknötchen in Verbindung standen, eine kleine längsgetroffene Vene, die von einer mehrfachen Lage von Geschwulstzellen umgeben war. Verfolgte man den Faden oder, wenn man will, den Stiel bis zu den Knötchen, so fand sich, dass das Gefäss sich verästelte, dass also derselbe Wachstumtypus nur in gesteigertem Masse beibehalten worden war. Dieser Befund ist eine gute Illustration zu der bekannten, besonders von R. Meyer ${ }^{1}$ ) immer wieder betonten Tatsache, dass die Sarkomzellen infolge der besseren Ernährung, die sie dort finden, sich mit Vorliebe der Gefässwand entlang schieben; ferner dazu, dass aus einer derartigen Anordnung nicht ohne weiteres auf einen Ausgang des Sarkoms von der Gefässwand geschlossen werden darf, wenn auch der Skeptizismus Meyers in Bezug auf die Adventitialsarkome (vergl. auch Kaufmann) verschiedentlich vielleicht $\mathrm{zu}$ weit geht. Es ist naheliegend, den erwähnten Wachstumsmodus auch zur Erklärung der Entstehung der eigentümlichen gröberen Stränge in Anspruch zu nehmen. Eine sichere Erklärung ihrer Entstehung kann ich nicht geben; vielleicht handelt es sich um vaskularisierte Adhäsionen, die von einem ihrer Haftpunkte abgerissen sind.

Ein Zweites, was auffiel, war die Cystenbildung. Wir fanden erstens verschieden grosse Cysten im Gebiet der einzeln entfernten soliden Geschwulstmassen (Fig. 1), zweitens solche im Gebiet eines membranösen Stücks (Fig. 2), endlich an der Oberfläche des Eierstockes (Fig. 6).

Das Aussehen und die Anordnung der Cysten liess anfangs teils an Lymphangiectasien bzw. Lymphangioma cysticum [( $\left.\mathrm{Sick}^{2}\right)$, Henke ${ }^{3}$ ), Nager ${ }^{4}$, Himmelheber $\left.{ }^{5}\right)$, teils an Peritonealcysten denken. Es war verführerisch, eine einheitliche Genese aller Cysten in dem einen oder anderen Sinne anzunehmen. Doch stellte sich bald heraus, dass dieser Gedanke fallen gelassen werden musste. Bei den erstgenannten Cysten fanden sich zumeist kreisrunde

1) R. Meyer, Das Sarcoma nteri. Veits Handb. d. Gyn. Bd. 3. I.

2) Sick, Beitrag zur Lehre vom Bau und Wachstum der Lymphangiome. Virchows Archiv. Bd. 170.

3) He nke, Multiples cystisches Lymphangiom der Bauchhöhle. Verhandl. d. deutschen path. Ges. Bd. 2.

4) Nager, Beitrag zur Kenntnis seltener Abdominaltumoren. Zieglers Beiträge. Bd. 36 .

5) Himmelbeber, Zur Kenntnis seltener cystischer Bildungen in der Bauchhöhle. Dieses Archiv. Bd. 87. 
Hohlräume, die gleichmässig geronnene Massen enthielten und von Sarkomzellen umrandet waren. Bei flüchtiger Betrachtung gewann man zunächst den Eindruck, als wäre stellenweise an der Innenwand eine gesonderte endotheliale Bekleidung nachweisbar. Weiter schien die peripher anliegende Zellschicht auf den ersten Blick eine gewisse Aehnlichkeit mit der zellreichen Theca int. eines Eierstockfollikels zu besitzen. Bei genauerem Studium erwies sich aber nicht nur, dass Endothel fehlte, sondern dass auch die Umrandung direkt von Sarkomzellen gebildet war, die zu schmalen Streifen geordnet verschiedentlich in das Lumen hineinragten. Es musste somit auf unechte oder Erweichungscysten, die durch Flüssigkeit abgerundet waren, geschlossen werden, und es liessen sich allmählich anch Uebergangsformen zwischen den obenerwähnten kleineren, unregelmässig geformten, zweifellos durch Zerstörung entstandenen Hohlräumen, in denen sich mitunter Blut, mitunter aber gleichfalls Gerinnungsmassen befanden, einerseits, andererseits den grossen rundlichen Räumen finden. Auf Grund des Ergebnisses der Thioninfärbung (Lilaton der Gerinnungsmassen) ist vielleicht mit partieller schleimiger Erweichung des Sarkomparenchyms oder des Grundgewebes zu rechnen. Doch haben sich dafür nirgends weitere bestimmte Anhaltspunkte ergeben. Vielmehr war von regressiven Metamorphosen mit Sicherheit nur eine ödematöse Durchtränkung zu konstatieren, wie eine solche auffallenderweise auch deutlich in dem weiter peripherisch von dem Cystenraum liegenden Geschwulstzellenring vorhanden war, während in dessen nächster Nachbarschaft die Sarkomelemente, wie gesagt, dichter angeordnet erschienen. Wir haben uns vielleicht vorzustellen, dass die dichtere Anordnung der Geschwulstzellen auf einer von den verflüssigten Partien ausgeübten Druckwirkung beruht.

Bei einer zweiten Art von Cysten liegen die Verhältnisse anders. Die in dem membranösen Gewebsstück gefundenen Cysten nnterscheiden sich sämtlich von den soeben beschriebenen dadurch, dass sie eine eigene Wandung besitzen. Einige können durch die darin enthaltene Muskulatur, durch das anhaftende Endothel und endlich durch den hämorrhagischen Inhalt ohne weiteres als Venen erkannt werden. Bei der Mehrzahl fehlt allerdings der genannte Inhalt. Muskulatur ist in der Umgebung nicht nachweisbar, dagegen hebt sich ein zweifelloses Endothel ab, so dass entweder an von der Oberfläche stammende abgeschlossene peritoneale Cystenräume oder an erweiterte Lymphgefässe gedacht werden muss. 
Der Nachweis kleinerer zweifelloser ektatischer Lymphgefässe, die Betrachtung aufeinanderfolgender Schnitte ergibt, dass hier die letztere Ansicht die richtige ist. Dagegen ist umgekehrt die Cyste an der Oberfläche des Ovariums zweifellos als Peritonealcyste zu deuten. Wie man sich erinnern wird (Fig. 6), erschien die Ovarialoberfläche mit einem deutlichen, niedrigen Epithel bekleidet, welches sich kontinuierlich auf die Innenfläche des Cystenraumes fortsetzte. In der Nachbarschaft war epithelbekleidetes Ligamentgewebe zu erkennen, in welchem übrigens erweiterte Lymphgefässe fehlen. Nach der ganzen Situation kann hier nur an eine Entstehung durch Verklebung des Epithels bzw. des Gewebes gedacht werden, und zwar dürfte, da nirgends Einsenkungen oder Abschnürungen des ersteren in dem Eierstocksgewebe wahrzunehmen sind, die Keimepithelgenese auszuschliessen sein.

Was nun in dem vorbeschriebenen Fall das grösste Interesse erweckt, sind die Beziehungen der Geschwulstzellen zu dem Endothel der Lymph- und Blutgefässe sowie des Peritoneums.

Nicht nur der kleinere Sarkomherd der letztgeschilderten Cyste (Fig. 6), nicht nur die in Fig. 2 wiedergegebenen, innerhalb von Gefässen liegenden, waren mit einem deutlichen, von. Tumorzellen ohne weiteres zu unterscheidenden Ueberzug platter Zellen versehen, sondern es liess sich auch ein gleichartiger Zellstrang inmitten des einen Geschwulstnestes konstatieren. Auch ohne Vergleich mit der Wandbekleidung in der Nachbarschaft konnten die Zellen als endotheliale bestimmt werden. Das formale Verhalten des Ueberzugs bezw. Streifens ist oben geschildert worden - es sei hier nur an der Hand der Figg. 3, 4, 5 noch einmal darauf hingewiesen, dass er im allgemeinen kontinuierlich, nur stellenweise lückenhaft ist, dass die Endothelien zumeist normal gefärbt, gut entwickelt, mitunter aber auch, besonders bei dem letzt erwähnten kleinen Cystenherd, blass und sehr niedrig erscheinen. Es sei endlich hinzugefügt, dass noch nachträglich in einer blutgefüllten (nicht abgebildeten) Vene ein Sarkomnest mit endothelialem Bezuge gefunden wurde.

In der Literatur sind nur wenige Angaben über ähnliche Erscheinungen zu finden. Borst gibt in einer ausführlichen Arbeit')

1) Borst, Das Verhalten der Endothelien bei der akuten und chronischen Entzündung sowie bei dem Wachstum der Geschwülste. Verhandl, d. phys.med. Ges. Würzburg. Bd. 31 . 
sowie in der "Geschwulstlehre"x) an, dass das Endothel der Gefässe in anliegende Carcinomnester hineinwachsen und dort durch Wucherung mit nachfolgender Bindegewebsbildung zur Bildung eines Stromas führen kann und M. B. Schmidt ${ }^{2}$ ) kommt zu dem Ergebnis, dass das Carcinom beim Durchbruch in das Gefäss bisweilen unterhalb des Endothels der Gefässwand fortwuchert, ohne dasselbe zu zerstören.

Aehnliches merkt auch Goldmann ${ }^{3}$ ) an mehreren Stellen seiner bekannten Arbeiten an. Im übrigen ist es mir nur gelungen, in einer Veröffentlichung von Knauer ${ }^{4}$ ) eine Mitteilung zu finden, welche fast vollständig auf das in Fig. 2 dargestellte Bild passt, aber es handelt sich hier um ein in einer Vene liegendes Leiomyomknötchen, und der Verf. geht auf die Entstehung und Bedeutung des Endothelüberzuges nicht ein.

Diese spärlichen Angaben, die sich fast ausschliesslich auf das Carcinom beziehen - nur Goldmann (l. c.) erwähnt gleichzeitig das Sarkom - müssen auffallen und es ist, wie ich glaube, nicht unangebracht, die Aufmerksamkeit der Autoren auf solche Befunde zu lenken.

Es muss festgelegt werden, dass das Gefässendothel, sowohl dasjenige der Blut- als auch der Lymphgefässe nicht nur widerstandsfähiger zu sein scheint, wie das gemeinhin angenommen wird, sondern dass es auch, und zwar nicht nur bei gutartigen Tumoren (Knauer), sondern auch bei exquisit bösartigen, und hier wieder bei Sarkomen (mein Fall), ebensogut wie bei Carcinomen (Borst) Proliferationserscheinungen zeigen kann.

M. B. Schmidt (1. c.) und Goldmann (l. c.) heben wesentlich nur die Widerstandsfähigkeit hervor, indem sie auf das erwähnte subendotheliale Geschwulstwachstum ${ }^{5}$ ) aufmerksam machen. Ich habe

1) Borst, Die Lehre von den Geschwülsten. Bd. I. S. 63 a.

2) M. B. Schmidt, Die Verbreitungswege der Carcinome und die Beziehung generalisierter Sarkome zu den leukämischen Neubildungen. Jena 1903.

3) Goldmann, Anatomische Untersuchungen über die Verbreitungswege dor bösartigen Gesohwülste. Bruns Beitr. z. klin. Chir. Bd. 18.

4) Knauer, Beitrag zur Anatomie der Uterusmyome. Chrobaks Beiträge zu Geb. u. Gyn.

5) Eine auf Anregung ron Prof. Borst nachträglich vorgenommene Weigert'sche Elastinfärbung in der.Absicht, zwischen dem Endothel und den Sarkomherden nach elastischen Fasern zu suchen und damit darzatun, dass das Endothel den Geschwolstmassen nicht unmittelbar aufsitze, hat ein negatives Resultat ergeben. 
dafür in meinem Fall, so sehr ich auch danach gesucht, keine ganz einwandsfreien Bilder, wie solche von den genannten Autoren gebracht worden sind, finden können. Doch dürfte in Fig. 6 bei dem kleinen, der Wand anliegenden Knötchen, umso mehr, als der peritoneale Endothelüberzug hier aus sehr niedrigen Zellen besteht und in weiteren Schnitten bald verschwindet, wohl an einen derartigen Vorgang gedacht werden. Obgleich der später an der Aussenfläche auftauchende, dem inneren gegenüber liegende (nicht abgebildete)' Herd (vergl. oben) direkt auf eine Durchbrechung der Cystenwand hinweist, so könnte das Endothel doch erhalten geblieben sein und es braucht nicht notwendigerweise eine wenigstens teilweise Neubildung angenommen zu werden, wie mir das zur Erklärung der Figg. 2-5 unerlässlich erscheint.

Bevor ich auf diese näher eingehe, sei noch bemerkt, dass bei der Peritonealcyste eine sekundäre Umschliessung vorher vorhandener Sarkomherde durch das Ligamentgewebe und etwa erst sekundär erfolgte Verklebung schwerlich in Betracht kommt; ferner, dass der zweite grössere Geschwulstherd (Fig. 6) sich entweder von dem wandständigen kleineren losgelöst hat oder von einem tatsächlich ausserdem vorhandenen stammen muss, der irgendwo die Cystenwand durchbrochen hat. Da letztere nicht ganz aufgeschnitten worden ist, so lässt sich darüber ein Urteil nicht fällen.

Bei den in den Lymphräumen und ganz analog auch in einer Vene (vergl. oben) gefundenen Sarkomherden kann nach der ganzen Lage der Dinge von einem nur subendothelialen Wachstum nicht die Rede sein. Es handelt sich hier um in das Lumen des Gefässes hineingewachsene, bzw. hineinragende Geschwulstknoten, die, wie die Figuren lehren, das eine Mal von verschiedenen Stellen der Wand herstammend, sich, nur durch den Endothelstreifen getrennt, dicht aneinander gelagert haben und entfernter von der Haftstelle frei im Lumen liegen. Hier ist meines Erachtens eine einfache passive Resistenz des sie bekleidenden Endothels unwahrscheinlich; vielmehr ist eine Neubildung von Elementen desselben vorauszusetzen. Zwar ist es mir nicht gelungen mitotische oder amitotische Kernfiguren zu entdecken. Aber darin liegt gewiss kein Gegengrund gegen die geäusserte Ansicht. Ausserdem spricht direkt für progressive Vorgänge der Umstand, dass die endothelialen Elemente, gleichwie wir das bei Zirkulationsstörungen, Entzündungen und Tumorbildung auch sonst sehen, vielfach geschwellt, vergrössert und epithelial erscheinen. Ich möchte bei dieser Gelegenheit auch 
an die Tatsache erinnern, dass z. B. das anfangs niedrige, endothelähnliche Epithel der Primordialfollikel sich erst mitotisch zu vermehren beginnt, wenn die Zellen höher geworden sind und richtig epitheliales Aussehen gewonnen haben.

Auf die Frage, ob das Endothel nur von den das Geschwulstknötchen überziehenden Elementen oder von der intakten Gefässwand herstammt, oder endlich, ob beide Vorgänge sich in dem Sinne kombinieren, dass von der Gefässwand Succurs geleistet wird, falls die Anbildung von Endothel an der Oberfläche des Knötchens mit dem Wachstum der Geschwulstzellen nicht gleichen Schritt hält, darauf ist zurzeit noch keine befriedigende Anwort zu geben. Fast sicher ist dagegen zu sagen, dass bei dem in Rede stehenden Vorgang wohl stets ein subendotheliales Wachsthum der Geschwulst vorausgegangen ist und keine Durchbrechung des Endothels an der Eintrittsstelle in das Gefäss stattgefunden hat. Anderenfalls wäre nämlich die völlige Abwesenheit der so oft beschriebenen und abgebildeten Thromben in der Umgebung der Geschwulstmassen kaum zu verstehen. Eine Thrombenbildung müsste erwartet werden, sobald Geschwulstmassen das schützende Endothel zerstört haben. Es ist vielleicht nicht überflüssig, hier noch zu betonen, dass die in Fig. 4 sichtbaren Lücken in dem Endothelüberzuge des Geschwulstknötchens als artefiziell entstanden angesehen werden müssen, da in den folgenden Schnitten solche Lücken nicht vorhanden waren.

Dass es sich bei Sarkomen und Carcinomen um ein im Prinzip gleiches Vorkommnis handelt, leuchtet ein. Und doch scheinen auch gewisse Unterschiede zu bestehen.

Herr Prof. Schottlaender ${ }^{1}$ ) hat mir Carcinompräparate gezeigt, bei denen sich in der Peripherie von Geschwulstnestern ein dentlicher, von den durch Druck abgeflachten Krebszellen wohl zu. unterscheidender, bisweilen syncytialer Endothelbelag befand; ausserdem solche, wo sich Endothelstränge zwischen die Krebszellen des Nestes hineinverfolgen liessen. Im wesentlichen handelt es sich hier wohl ganz um dasselbe, was Borst (s. o.) beschrieben hat, wenn es auch bisher nicht gelungen ist, spätere Stadien, welche auf Stromabildung hinweisen, zu entdecken.

1) Vgl. die demnächst erscheinende Monographie von Schottlaender und Kermaner. 
In meinem Fall konnte ich nirgends derartige fortgeschrittene Stadien des Prozesses nachweisen. Dass der Endothelstrang in Fig. 2 mit einer Einwucherung von Zellen in dem genannten Sinne nichts zu tun hat, wurde schon erwähnt.

Möglicherweise liegt der Grund für das unterschiedliche Verhalten von Sarkom und Carcinom in dieser Beziehung; in dem verschiedenen Aufbau und den verschiedenen Wachstumsbedingungen beider Geschwulstarten. Es muss, wie das besonders in Figur 4 deutlich ist, indessen durchweg auch sonst zu sehen war, hervorgehoben werden, dass das Gewebe der endothelial bekleideten Sarkomknötchen einen anderen Charakter besass, als das der übrigen Geschwulstherde. Es zeigte sich nämlich hier eine auffallend reichliche Zwischensubstanz, so reichlich, wie sie anderwärts nicht zu finden war. Man gelangt daher zu dem Wahrscheinlichkeitsschlusse, dass entweder die im Sinne einer verringerten Wucherungsfähigkeit veränderten Geschwulstzellen dem proliferierenden Endothel einen geringeren Widerstand entgegengesetzt haben, oder dass umgekehrt das wuchernde Endothel die entsprechende Veränderung des Sarkomgewebes verursacht hat. Eine Entscheidung lässt sich auch in dieser Frage nicht treffen. Wenn a priori zunächst manches dafür spricht, dass bei dem im gewissen Sinne bestehenden Antagonismus zwischen Endothel und Geschwulstgewebe, letzteres durch primäre Veränderung dem wuchernden Endothel die Bahn frei gibt, so ist auf der anderen Seite ein primärer Einfluss des durch irgend welchen Reiz in Wucherung geratenen Endothels auf das Geschwulstgewebe gewiss nicht von der Hand zu weisen. Vielleicht lässt sich in letzterem Sinne der Umstand verwerten, dass trotz intensiver Proliferation der Carcinomzellen (Goldmann, M. B. Schmidt) das Endothel erhalten bleiben kann, ferner, dass bei Einwucherung von Endothel in ein Krebsnest von einer besonderen Schädigung der Geschwulstzellen nichts bekannt gegeben worden ist.

\section{Erklärung der Abbildungen auf Tafel XI.}

Figur 1. Ein Stück des Sarcomgewebes mit Cysten und verschiedenen Ausläufern. (Normale Grösse.)

Figur 2. Schnitt durch die Cysten der Membran. Die Geschwulstknoten sind mit Endothel bekleidet. (Vergrösserung $31: 1$, Endothel mit etwas stärkerer Vergrösserung eingezeichnet.) 
316 Goldenstein, Cystisches Sarkom des Beckenperitoneums.

Figur 3. Ein Teil des im Lymphgefäss freiliegenden Sarkomknotens (siehe Fig. 2) mit Endothel auf der Oberfläche. (Vergrösserung $250: 1$. )

Figur 4. Ein Teil desselben (Fig. 2 and 3) Sarkomknotens mit Endothel auf der Oberfläche und gegenüberliegenden endothelbekleideten Gefässwand. (Vergrösserung $250: 1$.)

Figur 5. Durchbruchstelle des in Figg. 2, 3 and 4 abgebildeten Knotens in das Lymphgefäss. Der Knoten ist mit Endothel bekleidet. (Vergr. $62: 1$.).

Figur 6. Rest des Ovariums mit aufsitzender Verklebungscyste. Das der Cystenwand aufsitzende Sarkom ist mit Endothel bekleidet (auf dem Bild nicht zu sehen). Vergrösserung 4:1. 
હู่
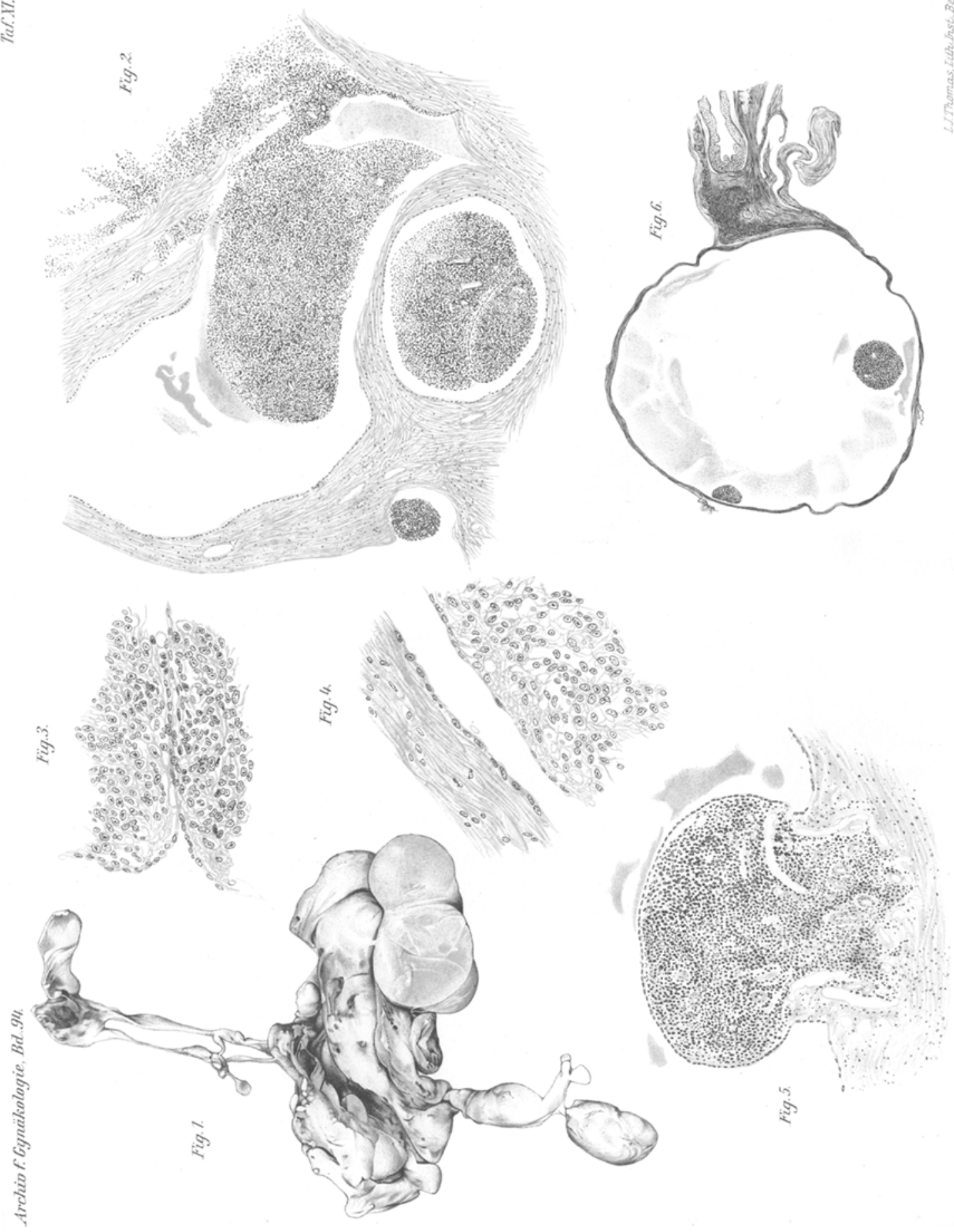